\title{
Optimal allocation of measurements in a linear calibration process
}

\author{
Tsai-Yu Lin and Chen-Tuo Liao \\ Division of Biometry, Institute of Agronomy, National Taiwan University, Taipei, Taiwan, 106, \\ R.O.C. (E-mail: d89621201@ntu.edu.tw, ctliao@ntu.edu.tw)
}

Received October 2003

\begin{abstract}
A problem of allocation of measurements for a linear calibration process is considered in this article. It is assumed that a total of $N$ measurements are made some of which may be measurements on two distinct standards, while the remaining measurements are on $m$ different unknown specimens. We discuss allocation of the $N$ measurements for the two standards and $m$ unknown specimens based on A-optimality criterion, which is applied to asymptotic variances of maximum likelihood estimators for the true values of unknown specimens. It can be shown that the optimal allocation depends on the true values of unknown specimens. Hence, the user may resort to locally or Bayesian A-optimal measurement designs. Some practical solution is presented. Furthermore, the impact of prior on the allocation is also discussed.
\end{abstract}

Key words: Calibration model, A-optimality criterion, measurement design, Bayesian design.

\section{Introduction}

A measurement process is typically subject to errors which are generally classified as systematic, random and a combination of both. Random errors are defined to have a zero expected value and systematic errors are defined to be due to biases in the measurement process. It is very common that the instrument is considered to have a linear systematic error in which the measured value of an unknown specimen ("unknown" for short) is described by the model

$$
y_{i}=\alpha+\beta \tau+\epsilon_{i},
$$

where $\alpha$ and $\beta$ are parameters of the systematic error; $\tau$ represents the true value of the unknown being measured; and $\epsilon_{i}$ represents the random error. 
Since "standards" have known true values, the errors associated with the measurement process are observed whenever a standard is measured. Therefore, the known standards are always used to estimate parameters of the errors.

The calibration problem for estimation procedure has been extensively studied. The main results can be found in Fuller (1987) and Brown (1993). Literature pertaining to calibration problems involving allocation of the measurements appears to begin with Pepper (1973). He discussed measurement designs for the process described by an additive model of the form $y_{i}=\tau+\left(b_{i}+\eta_{i}\right)$, where the quantity $\left(b_{i}+\eta_{i}\right)$ represents the random error. $b_{i}$ 's arise from a random walk process and $\eta_{i}$ 's are identical and independent normal random variables with common mean 0 and variance $\sigma_{\eta}^{2}$. Perng and Tong (1977) considered the linear calibration model of (1.1). They presented a sequential procedure, observing either standard or unknown at each time, for optimal allocation of measurements for interval estimation of one single unknown so that coverage probability is maximized. More recently, Liao, Taylor and Iyer (2000) discussed optimum balanced designs for the simple additive model $y_{i}=\tau+e_{i}$, where $e_{i}$ 's arise from a first order autoregressive process $(\mathrm{AR}(1))$.

As in many practical situations, the linear calibration model of (1.1) is required to estimate the actual concentration of an analyte in a specimen for a given response in various measurement processes. One of the examples described in Hunter and Lamboy (1981) is given below.

Example 1.1. A chemist wants to establish a calibration line to use in measuring the amount of molybdenum in specimens sent to an analytical laboratory. Table 1.1 is the real data.

Figure 1.1. gives a plot of the known amount of molybdenum against the measured amount of molybdenum, which is quite linear on this scale.

We thus consider the linear calibration model of (1.1) with random errors being assumed to be identical and independent normal random variables with common mean 0 and variance $\sigma^{2}$. Note that the normality of the random errors is in practice a reasonable assumption. It is well known that variance of LSE (least square estimator) of slope parameter in the simple regression model is minimized by putting equal proportion of trials at the two end points of the design region. See Atkinson and Donev (1992). Therefore, for both practical and theoretical considerations, we focus on the optimal allocation of $N$ measurements for two standards and $m$ unknowns in this study. The following example of a practical situation provides a setting to motivate this study.

Table 1.1. The real data for the calibration process of the amount of molybdenum

\begin{tabular}{lrllllllllr}
\hline The known amount of molybdenum & 1.0 & 1.0 & 2.0 & 2.0 & 3.0 & 3.0 & 4.0 & 4.0 & 5.0 & 5.0 \\
The measured amount of molybdenum & 1.8 & 1.6 & 3.1 & 2.6 & 3.6 & 3.4 & 4.9 & 4.2 & 6.0 & 5.9 \\
The known amount of molybdenum & 6.0 & 6.0 & 7.0 & 7.0 & 8.0 & 8.0 & 9.0 & 9.0 & 10.0 & 10.0 \\
The measured amount of molybdenum & 6.8 & 6.9 & 8.2 & 7.3 & 8.8 & 8.5 & 9.5 & 9.5 & 10.6 & 10.6 \\
\hline
\end{tabular}




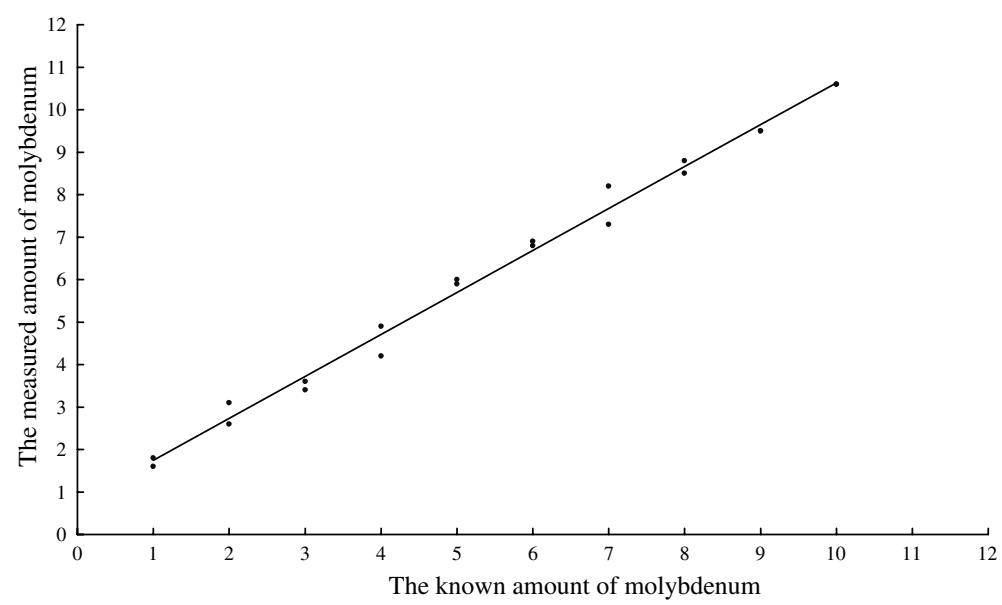

Fig. 1.1. The known amount of molybdenum versus the measured amount of molybdenum. The points represent the measured values, the solid line is the linear calibration model estimated by least square estimation

Example 1.2 A chemistry laboratory may be requested to analyze water samples from different sources. The small quantity of some toxic substance like cadmium in the water samples is highly concerned. Suppose for the used measurement instrument AAS (Atomic Absorption Spectroscopy), a chemist knows that the relationship between $\tau$, the actual amount of a toxic substance (concentration of cadmium) present in water samples and $y_{i}$, the measured value (absorption) is given by the regression function described by equation (1.1). Typically, a set of standard solutions with predetermined concentrations can be prepared in order to estimate the parameters associated with the errors of measurement process. Before conducting the measurements, the chemist needs a "good" approach to allocating standards and unknown specimens from different sources within a fixed total number of measurements.

In the next section, we formulate the problem of interest. In Section 3, we present maximum likelihood estimation for the parameters in model (1.1). Section 4 provides some practical locally A-optimal measurement designs. Section 5 investigates Bayesian A-optimal measurement designs and the impact of prior on the designs.

\section{The problem of interest}

Let the number of measurements on two distinct standards $S_{0}$ and $S_{1}$ be denoted by $a_{0}$ and $a_{1}$, respectively, and the number of measurements on unknown $U_{j}$ is denoted by $n_{j}$, for $j=1,2, \ldots, m$. Thus, the total number of measurements is $N=a_{0}+a_{1}+\sum_{j=1}^{m} n_{j}$. Moreover, let $x_{0}$ and $x_{1}$ be the known true values of standards $S_{0}$ and $S_{1}$, respectively, and $\tau_{j}$ denote the true value of unknown $U_{j}$, for $j=1,2, \ldots, m$. Define indicator functions $\delta_{i}^{S_{k}}$ and $\delta_{i}^{U_{j}}$ by 
$\delta_{i}^{S_{k}}= \begin{cases}1 & \text { if } k \in\{0,1\} \text { and observation } i \text { is of standard } S_{k}, \\ 0 & \text { otherwise, }\end{cases}$

and

$$
\delta_{i}^{U_{j}}= \begin{cases}1 & \text { if } j \in\{1,2, \ldots, m\} \text { and observation } i \text { is of unknown } U_{j}, \\ 0 & \text { otherwise. }\end{cases}
$$

Then, model (1.1) can be rewritten as

$$
y_{i}=\alpha+\beta\left(\delta_{i}^{S_{0}} x_{0}+\delta_{i}^{S_{1}} x_{1}+\sum_{j=1}^{m} \delta_{i}^{U_{j}} \tau_{j}\right)+\epsilon_{i}, \quad \text { for } i=1,2, \ldots, N .
$$

The random errors $\epsilon_{i}$ 's are now assumed to be independent and identically distributed with $N\left(0, \sigma^{2}\right)$. This paper is concerned with obtaining optimal allocation of $S_{0}, S_{1}, U_{1}, U_{2}, \ldots, U_{m}$ such that $\tau_{1}, \tau_{2}, \ldots, \tau_{m}$ can be estimated most efficiently. In particular, we consider A-optimality criterion according to which the average asymptotic variance of MLEs of $\tau_{j}$ 's is minimized.

\section{Maximum likelihood estimation}

The log-likelihood function corresponding to the observed data of (2.1) is given by

$$
l=-\frac{N}{2} \log (2 \pi)-\frac{N}{2} \log \left(\sigma^{2}\right)-\frac{1}{2 \sigma^{2}} \sum_{i=1}^{N}\left[y_{i}-\alpha-\beta\left(\delta_{i}^{S_{0}} x_{0}+\delta_{i}^{S_{1}} x_{1}+\sum_{j=1}^{m} \delta_{i}^{U_{j}} \tau_{j}\right)\right]^{2},
$$

from which the following likelihood estimators are derived.

$$
\begin{aligned}
& \hat{\alpha}=\bar{y}-\hat{\beta} \bar{x}, \\
& \hat{\beta}=\frac{\sum_{i=1}^{N} \delta_{i}^{S_{0}}\left(y_{i}-\bar{y}\right)\left(x_{0}-\bar{x}\right)+\sum_{i=1}^{N} \delta_{i}^{S_{1}}\left(y_{i}-\bar{y}\right)\left(x_{1}-\bar{x}\right)}{a_{0}\left(x_{0}-\bar{x}\right)^{2}+a_{1}\left(x_{1}-\bar{x}\right)^{2}}, \\
& \hat{\tau}_{j}=\frac{\bar{y}_{j}-\hat{\alpha}}{\hat{\beta}}, \quad \text { for } j=1,2, \ldots, m,
\end{aligned}
$$

where $\bar{y}=\frac{1}{a_{0}+a_{1}}\left(\sum_{i=1}^{N} \delta_{i}^{S_{0}} y_{i}+\sum_{i=1}^{N} \delta_{i}^{S_{1}} y_{i}\right), \bar{x}=\frac{a_{0} x_{0}+a_{1} x_{1}}{a_{0}+a_{1}}$ and $\bar{y}_{j}=\frac{1}{n_{j}} \sum_{i=1}^{N} \delta_{i}^{U_{j}} y_{i}$, for $j=1,2, \ldots, m$.

Clearly, the MLE for $\tau_{j}$ given in (3.4) is the well known classical estimator computed from $\hat{\alpha}$ of (3.2) and $\hat{\beta}$ of (3.3), which only depend on the observed data of $S_{0}$ and $S_{1}$.

Computing the second derivatives of log-likelihood function (3.1) with respect to parameters $\alpha, \beta, \tau_{1}, \tau_{2}, \ldots, \tau_{m}, \sigma^{2}$ and taking expectation, we can obtain the Fisher information matrix. By using the standard properties of partitioned matrices (Graybill, 1983), we then invert the Fisher information to obtain the asymptotic covariance matrix of MLEs for $\tau_{j}$ 's, which are of primary interest, as follows. 


$$
\operatorname{Var}(\hat{\tau})=\left\{v_{j k}\right\}, j=1,2, \ldots, m ; k=1,2, \ldots, m,
$$

where $\hat{\tau}=\left[\hat{\tau}_{1}, \hat{\tau}_{2}, \ldots, \hat{\tau}_{m}\right]^{\prime}$ and

$$
v_{j k}= \begin{cases}\frac{\sigma^{2}}{\beta^{2}}\left[\frac{1}{n_{j}}+\frac{a_{0}\left(x_{0}-\tau_{j}\right)^{2}+a_{1}\left(x_{1}-\tau_{j}\right)^{2}}{a_{0} a_{1}\left(x_{0}-x_{1}\right)^{2}}\right] & \text { if } \mathrm{j}=\mathrm{k}, \\ \frac{\sigma^{2}}{\beta^{2}}\left[\frac{a_{0}\left(x_{0}-\tau_{j}\right)\left(x_{0}-\tau_{k}\right)+a_{1}\left(x_{1}-\tau_{j}\right)\left(x_{1}-\tau_{k}\right)}{a_{0} a_{1}\left(x_{0}-x_{1}\right)^{2}}\right] & \text { if } \mathrm{j} \neq \mathrm{k} .\end{cases}
$$

The details of the derivation are given in Appendix.

\section{Locally A-optimal measurement design}

It is easy to see that an explicit expression for the trace of covariance matrix $\operatorname{Var}(\hat{\tau})$ is given by

$$
\operatorname{Trace}[\operatorname{Var}(\hat{\tau})]=\frac{\sigma^{2}}{\beta^{2}}\left[\sum_{j=1}^{m} \frac{1}{n_{j}}+\frac{1}{a_{1}} \sum_{j=1}^{m} \frac{\left(x_{0}-\tau_{j}\right)^{2}}{\left(x_{0}-x_{1}\right)^{2}}+\frac{1}{a_{0}} \sum_{j=1}^{m} \frac{\left(x_{1}-\tau_{j}\right)^{2}}{\left(x_{0}-x_{1}\right)^{2}}\right] .
$$

Let $\theta_{0}=\sum_{j=1}^{m} \frac{\left(x_{0}-\tau_{j}\right)^{2}}{\left(x_{0}-x_{1}\right)^{2}}$ and $\theta_{1}=\sum_{j=1}^{m} \frac{\left(x_{1}-\tau_{j}\right)^{2}}{\left(x_{0}-x_{1}\right)^{2}}$. Also let $b_{0}=a_{0} / N, b_{1}=a_{1} / N$ and $r_{j}=n_{j} / N$, for $j=1,2, \ldots, m$. To obtain A-optimal measurement designs for estimating $\tau_{j}$ 's, we need to minimize the following objective function

$$
f\left(b_{0}, b_{1}, r_{1}, r_{2}, \ldots, r_{m}, \theta_{0}, \theta_{1}\right)=\frac{\theta_{1}}{b_{0}}+\frac{\theta_{0}}{b_{1}}+\sum_{j=1}^{m} \frac{1}{r_{j}},
$$

subject to the constraint

$$
b_{0}+b_{1}+\sum_{j=1}^{m} r_{j}=1
$$

For given values of $\theta_{0}$ and $\theta_{1}$, by using a Lagrange multiplier, we have the following optimal allocation of measurements for the standards and unknowns

$$
\begin{aligned}
& b_{0}^{*}=\frac{\sqrt{\theta_{1}}}{\sqrt{\theta_{0}}+\sqrt{\theta_{1}}+m}, \\
& b_{1}^{*}=\frac{\sqrt{\theta_{0}}}{\sqrt{\theta_{0}}+\sqrt{\theta_{1}}+m}
\end{aligned}
$$

and

$$
r_{j}^{*}=\frac{1}{\sqrt{\theta_{0}}+\sqrt{\theta_{1}}+m}, \quad \text { for } j=1,2, \ldots, m .
$$

Clearly, the values of $b_{0}^{*}, b_{1}^{*}, r_{1}^{*}, r_{2}^{*}, \ldots, r_{m}^{*}$ depend on parameters $\tau_{1}, \tau_{2}, \ldots, \tau_{m}$. Hence, the problem of allocation cannot be solved unless one has prior information on these parameters. We first consider the following strategy so as to obtain a locally A-optimal measurement design. In practice, according to knowledge of the user, it may be possible to designate standards $S_{0}$ and $S_{1}$ such that the range $\left[x_{0}, x_{1}\right]$ is large enough to cover the true values of unknowns. Then a very natural estimate for $\tau_{j}$ is the average of $x_{0}$ and $x_{1}$, i.e. letting 
Table 4.1. The locally A-optimal measurement designs for $\tau_{j}=\left(x_{0}+x_{1}\right) / 2$, for $j=1,2, \ldots, m$; and $1 \leq m \leq 5$

\begin{tabular}{lll}
\hline $\mathrm{m}$ & $b^{*}$ & $r_{j}^{*}$ \\
\hline 1 & 0.250 & 0.500 \\
2 & 0.207 & 0.293 \\
3 & 0.183 & 0.211 \\
4 & 0.167 & 0.167 \\
5 & 0.155 & 0.138 \\
\hline
\end{tabular}

$\tau_{j}=\frac{x_{0}+x_{1}}{2}, \quad$ for $j=1,2, \ldots, m$.

Substituting the values of (4.3) in $\theta_{0}$ and $\theta_{1}$, we have $\theta_{0}=\theta_{1}=m / 4$. Thus, for fixed $m$, the values of $b_{0}^{*}, b_{1}^{*}$ and $r_{j}^{*}$ can be determined. Table 4.1 displays some locally A-optimal measurement designs based on this strategy. Note that we use $b^{*}$ to denote both $b_{0}^{*}$ and $b_{1}^{*}$ in the table since their values are equal.

To evaluate how good the locally A-optimal designs of Table 4.1 are in estimating the true values of unknowns, the following simulation study is carried out. In the simulation study, we consider the two cases that $m=1$ and $m=2$. The values of parameters are set as follows. $x_{0}=0, x_{1}=1, \alpha=0.2$, $\beta=0.4$ and $\sigma=0.1 ; N=20,40,60,80,100$; for the case that $m=1, \tau_{1}$ ranges from 0.1 to 1.5 in steps of 0.2 ; for the case that $m=2$, the pair of values $\left(\tau_{1}, \tau_{2}\right)$ range from 0.25 to 1.5 in steps of 0.2 . Since the classical estimator of $\tau_{j}$ does not have a finite expectation or a finite variance, the median and interquartile range (IQR) of the estimated $\tau_{j}$ 's are reported from 5000 simulated data sets for each given parameter combination. Table 4.2 displays the simulation results for the case that $m=1$.

It has shown that the locally A-optimal measurement design performs very satisfactory accuracy in estimation, based on the reported medians, for all the cases considered even the value of $\tau_{1}$ occurs outside the range $\left[x_{0}, x_{1}\right]$. But it may only perform great precision in estimation, based on the reported IQRs, for the large sample size cases with $N \geq 40$. Notice that the minimum

Table 4.2. Simulation results for the case that $m=1$

\begin{tabular}{lllllll}
\hline$\tau_{1}$ & & $N=20$ & $N=40$ & $N=60$ & $N=80$ & $N=100$ \\
\hline 0.1 & Median & 0.1002 & 0.1014 & 0.0991 & 0.1004 & 0.1002 \\
& IQR & 0.1720 & 0.1262 & 0.1003 & 0.0868 & 0.0775 \\
0.3 & Median & 0.3002 & 0.2996 & 0.2991 & 0.3011 & 0.3002 \\
& IQR & 0.1586 & 0.1082 & 0.0896 & 0.0778 & 0.0716 \\
0.5 & Median & 0.4995 & 0.5000 & 0.4987 & 0.5006 & 0.5016 \\
& IQR & 0.1517 & 0.1080 & 0.0869 & 0.0759 & 0.0673 \\
0.7 & Median & 0.7003 & 0.7010 & 0.6985 & 0.6994 & 0.7003 \\
& IQR & 0.1578 & 0.1132 & 0.0931 & 0.0796 & 0.0711 \\
0.9 & Median & 0.9019 & 0.8965 & 0.8991 & 0.9011 & 0.8990 \\
& IQR & 0.1747 & 0.1226 & 0.1007 & 0.0881 & 0.0779 \\
1.1 & Median & 1.1023 & 1.0981 & 1.1036 & 1.1027 & 1.0998 \\
& IQR & 0.1954 & 0.1392 & 0.1173 & 0.0989 & 0.0896 \\
1.3 & Median & 1.3019 & 1.3023 & 1.3004 & 1.3001 & 1.3005 \\
& IQR & 0.2325 & 0.1663 & 0.1320 & 0.1142 & 0.1037 \\
1.5 & Median & 1.5024 & 1.4972 & 1.4979 & 1.4993 & 1.5016 \\
& IQR & 0.2697 & 0.1824 & 0.1484 & 0.1281 & 0.1202 \\
\hline
\end{tabular}




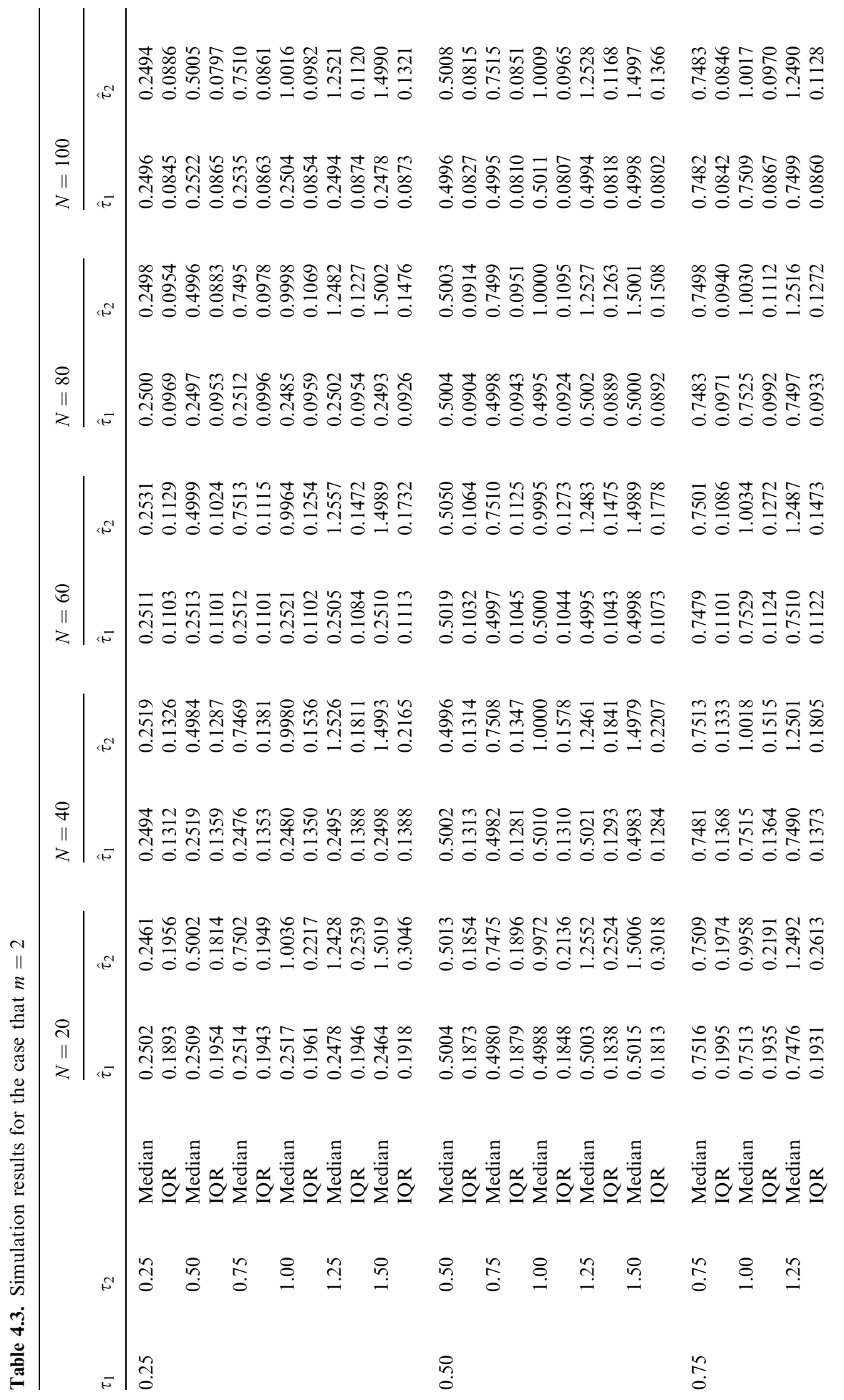




\begin{tabular}{|c|c|c|c|c|c|}
\hline \multirow[b]{2}{*}{$\begin{array}{l}8 \\
\frac{8}{11} \\
z\end{array}$} & $\langle\vec{N}$ & 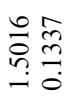 & 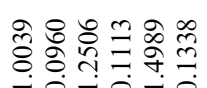 & 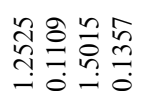 & 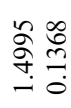 \\
\hline & $\vec{N}$ & $\begin{array}{l}\frac{0}{0} \\
\stackrel{0}{0} \\
\stackrel{0}{0}\end{array}$ & 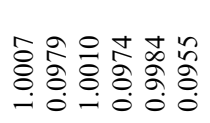 & 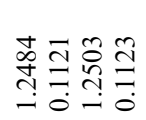 & 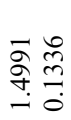 \\
\hline & «N & 尽 & 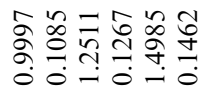 & 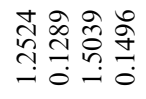 & 咅跡 \\
\hline $\begin{array}{l}\infty \\
11 \\
z\end{array}$ & $\vec{k}$ & 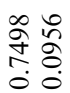 & 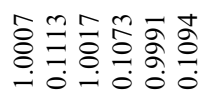 & 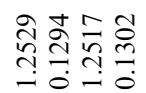 & 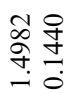 \\
\hline & « & 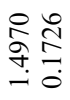 & 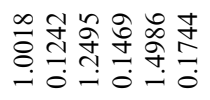 & 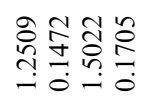 & 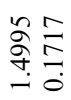 \\
\hline $\begin{array}{l}8 \\
11 \\
z\end{array}$ & $\vec{N}$ & $\begin{array}{l}\vec{\infty} \\
\stackrel{\infty}{+} \stackrel{\infty}{=} \\
\stackrel{0}{0}\end{array}$ & 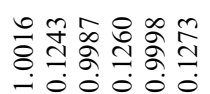 & 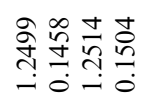 & 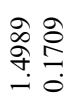 \\
\hline & $\langle\sim$ & 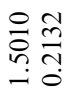 & 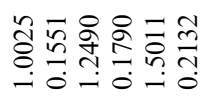 & 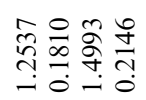 & 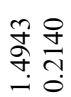 \\
\hline $\begin{array}{l}\stackrel{8}{+} \\
\text { II } \\
z\end{array}$ & $\vec{\omega}$ & 离考 & 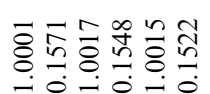 & 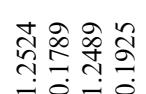 & 容守 \\
\hline & $\stackrel{\leftrightarrow}{N}$ & 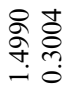 & 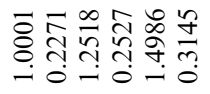 & 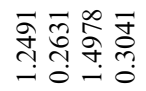 & 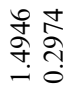 \\
\hline $\begin{array}{l}\stackrel{\overbrace{}}{N} \\
11 \\
z\end{array}$ & $\vec{N}$ & 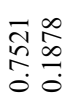 & 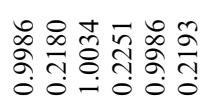 & 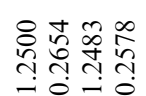 & 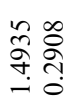 \\
\hline & & 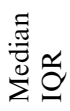 & 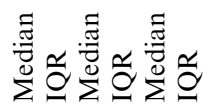 & 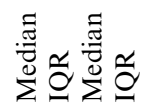 & 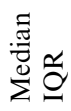 \\
\hline & ה & $\stackrel{?}{i}$ & $\underset{i}{\stackrel{n}{r}} \stackrel{n}{n}$ & $\stackrel{n}{\dddot{n}} \stackrel{n}{n}$ & $\stackrel{?}{?}$ \\
\hline & $\vec{N}$ & & $\stackrel{8}{8}$ & $\stackrel{n}{\sim}$ & $\stackrel{\text { n̊ }}{n}$ \\
\hline
\end{tabular}


IQR occurs at $\tau_{1}=0.5$ for any $N$, this is because that the used design is the locally A-optimal design for $\tau_{1}=0.5$. However, for any given $N$, the design proposed is likely to be robust to the value of $\tau_{1}$ provided it is not much larger than $x_{1}$. Therefore, when there is no prior information available on the true value of unknown, the design proposed can be a practical choice. Similar results can be found for the case that $m=2$ in Table 4.3.

\section{Bayesian A-optimal measurement design}

It is well known that optimal designs for non-linear models like model (2.1) depend on the values of unknown parameters. In this section, instead of locally A-optimal designs, a prior for $\tau_{1}, \tau_{2}, \ldots, \tau_{m}$ will be incorporated into the objective function of (4.1) to yield Bayesian A-optimal designs. We refer Atkinson and Donev (1992) for a general theory regarding the Bayesian design.

It is assumed that $\tau_{j}$ 's are uncorrelated random variables with common and known mean $x_{\tau}$ and variance $s_{\tau}^{2}$. Notice that only the first and second moments of $\tau_{j}$ 's are required, there is no specified distribution. Then the objective function for the allocation obtained from taking expectation of (4.1) over this prior is given by

$$
\begin{aligned}
E_{\theta_{0}, \theta_{1}}\left[f\left(b_{0}, b_{1}, r_{1}, r_{2}, \ldots, r_{m}, \theta_{0}, \theta_{1}\right)\right]= & \frac{m\left[\left(x_{1}-x_{\tau}\right)^{2}+s_{\tau}^{2}\right]}{b_{0}\left(x_{0}-x_{1}\right)^{2}} \\
& +\frac{m\left[\left(x_{0}-x_{\tau}\right)^{2}+s_{\tau}^{2}\right]}{b_{1}\left(x_{0}-x_{1}\right)^{2}}+\sum_{j=1}^{m} \frac{1}{r_{j} .}
\end{aligned}
$$

Similarly, under the constraint of (4.2), we have the following optimal allocation of measurements for the standards and unknowns

$$
\begin{aligned}
& b_{0}^{*}=\frac{1}{\sqrt{\frac{\left(x_{0}-x_{\tau}\right)^{2}+s_{\tau}^{2}}{\left(x_{1}-x_{\tau}\right)^{2}+s_{\tau}^{2}}}+\sqrt{\frac{m\left(x_{0}-x_{1}\right)^{2}}{\left(x_{1}-x_{\tau}\right)^{2}+s_{\tau}^{2}}}+1}, \\
& b_{1}^{*}=\frac{1}{\sqrt{\frac{\left(x_{1}-x_{\tau}\right)^{2}+s_{\tau}^{2}}{\left(x_{0}-x_{\tau}\right)^{2}+s_{\tau}^{2}}}+\sqrt{\frac{m\left(x_{0}-x_{1}\right)^{2}}{\left(x_{0}-x_{\tau}\right)^{2}+s_{\tau}^{2}}}+1}
\end{aligned}
$$

and

$$
r_{j}^{*}=\frac{1}{\sqrt{\frac{m\left[\left(x_{0}-x_{\tau}\right)^{2}+s_{\tau}^{2}\right]}{\left(x_{0}-x_{1}\right)^{2}}+\sqrt{\frac{m\left[\left(x_{1}-x_{\tau}\right)^{2}+s_{\tau}^{2}\right]}{\left(x_{0}-x_{1}\right)^{2}}}+m}}, \text { for } j=1,2, \ldots, m .
$$

To investigate how the prior affects the allocation, we calculate $b_{0}^{*}, b_{1}^{*}$ and $r_{j}^{*}$ for some given values of $x_{\tau}$ and $s_{\tau}$. Without loss of generality, let $x_{0}=0$ and $x_{1}=1$. The results are displayed in Table 5.1.

It is of interest to see that the allocation of standards $S_{0}$ and $S_{1}$ is highly related to $x_{\tau}$. For a fixed $s_{\tau}$, the value of $b_{0}^{*}\left(b_{1}^{*}\right)$ increases as the distance between $x_{\tau}$ and $x_{0}\left(x_{1}\right)$ decreases. This is fairly reasonable because the user would like to give more weight to the standard whose value may be closer to 
Table 5.1. Bayesian A-optimal measurement designs for $x_{\tau}$ being from 0.1 to 1.7 in steps of $0.2 ; s_{\tau}$ from 0.1 to 0.5 in steps of 0.2 ; and $1 \leq m \leq 3$

\begin{tabular}{|c|c|c|c|c|c|c|c|c|c|c|c|c|c|c|c|}
\hline$x_{\tau}$ & $s_{\tau}$ & $m$ & $b_{0}^{*}$ & $b_{1}^{*}$ & $r_{j}^{*}$ & $x_{\tau}$ & $s_{\tau}$ & $b_{0}^{*}$ & $b_{1}^{*}$ & $r_{j}^{*}$ & $x_{\tau}$ & $s_{\tau}$ & $b_{0}^{*}$ & $b_{1}^{*}$ & $r_{j}^{*}$ \\
\hline \multirow[t]{9}{*}{0.1} & \multirow[t]{3}{*}{0.1} & 1 & 0.442 & 0.069 & 0.489 & \multirow[t]{9}{*}{0.3} & \multirow[t]{3}{*}{0.1} & 0.349 & 0.156 & 0.494 & \multirow[t]{9}{*}{0.5} & \multirow[t]{3}{*}{0.1} & 0.252 & 0.252 & 0.495 \\
\hline & & 2 & 0.368 & 0.057 & 0.287 & & & 0.290 & 0.130 & 0.290 & & & 0.209 & 0.209 & 0.291 \\
\hline & & 3 & 0.326 & 0.051 & 0.208 & & & 0.257 & 0.115 & 0.210 & & & 0.185 & 0.185 & 0.210 \\
\hline & \multirow[t]{3}{*}{0.3} & 1 & 0.419 & 0.140 & 0.442 & & \multirow[t]{3}{*}{0.3} & 0.348 & 0.194 & 0.457 & & \multirow[t]{3}{*}{0.3} & 0.269 & 0.269 & 0.462 \\
\hline & & 2 & 0.354 & 0.118 & 0.264 & & & 0.293 & 0.163 & 0.272 & & & 0.226 & 0.226 & 0.274 \\
\hline & & 3 & 0.317 & 0.106 & 0.193 & & & 0.261 & 0.145 & 0.198 & & & 0.201 & 0.201 & 0.199 \\
\hline & \multirow[t]{3}{*}{0.5} & 1 & 0.405 & 0.201 & 0.394 & & \multirow[t]{3}{*}{0.5} & 0.352 & 0.239 & 0.409 & & \multirow[t]{3}{*}{0.5} & 0.293 & 0.293 & 0.414 \\
\hline & & 2 & 0.349 & 0.173 & 0.239 & & & 0.301 & 0.204 & 0.247 & & & 0.250 & 0.250 & 0.250 \\
\hline & & 3 & 0.315 & 0.156 & 0.176 & & & 0.271 & 0.184 & 0.182 & & & 0.225 & 0.225 & 0.184 \\
\hline \multirow[t]{9}{*}{0.7} & \multirow[t]{3}{*}{0.1} & 1 & 0.156 & 0.349 & 0.494 & \multirow[t]{9}{*}{0.9} & \multirow[t]{3}{*}{0.1} & 0.069 & 0.442 & 0.489 & \multirow[t]{9}{*}{1.1} & \multirow[t]{3}{*}{0.1} & 0.063 & 0.492 & 0.445 \\
\hline & & 2 & 0.130 & 0.290 & 0.290 & & & 0.057 & 0.368 & 0.287 & & & 0.053 & 0.415 & 0.266 \\
\hline & & 3 & 0.115 & 0.257 & 0.210 & & & 0.051 & 0.326 & 0.208 & & & 0.047 & 0.371 & 0.194 \\
\hline & \multirow[t]{3}{*}{0.3} & 1 & 0.194 & 0.348 & 0.457 & & \multirow[t]{3}{*}{0.3} & 0.140 & 0.419 & 0.442 & & \multirow[t]{3}{*}{0.3} & 0.129 & 0.464 & 0.407 \\
\hline & & 2 & 0.163 & 0.293 & 0.272 & & & 0.118 & 0.354 & 0.264 & & & 0.110 & 0.397 & 0.246 \\
\hline & & 3 & 0.145 & 0.261 & 0.198 & & & 0.106 & 0.317 & 0.193 & & & 0.099 & 0.358 & 0.181 \\
\hline & \multirow[t]{3}{*}{0.5} & 1 & 0.239 & 0.352 & 0.409 & & \multirow[t]{3}{*}{0.5} & 0.201 & 0.405 & 0.394 & & \multirow[t]{3}{*}{0.5} & 0.188 & 0.445 & 0.368 \\
\hline & & 2 & 0.204 & 0.301 & 0.247 & & & 0.173 & 0.349 & 0.239 & & & 0.163 & 0.386 & 0.226 \\
\hline & & 3 & 0.184 & 0.271 & 0.182 & & & 0.156 & 0.315 & 0.176 & & & 0.148 & 0.350 & 0.167 \\
\hline \multirow[t]{9}{*}{1.3} & \multirow[t]{3}{*}{0.1} & 1 & 0.121 & 0.498 & 0.382 & \multirow[t]{9}{*}{1.5} & 0.1 & 0.169 & 0.499 & 0.332 & 1.7 & 0.1 & 0.207 & 0.499 & 0.293 \\
\hline & & 2 & 0.104 & 0.430 & 0.233 & & & 0.149 & 0.439 & 0.206 & & & 0.185 & 0.445 & 0.185 \\
\hline & & 3 & 0.094 & 0.389 & 0.172 & & & 0.136 & 0.401 & 0.154 & & & 0.171 & 0.411 & 0.139 \\
\hline & 0.3 & 1 & 0.154 & 0.484 & 0.363 & & 0.3 & 0.187 & 0.491 & 0.321 & & 0.3 & 0.218 & 0.495 & 0.287 \\
\hline & & 2 & 0.134 & 0.421 & 0.223 & & & 0.165 & 0.434 & 0.200 & & & 0.195 & 0.442 & 0.181 \\
\hline & & 3 & 0.122 & 0.382 & 0.165 & & & 0.152 & 0.398 & 0.150 & & & 0.180 & 0.409 & 0.137 \\
\hline & 0.5 & 1 & 0.196 & 0.468 & 0.336 & & 0.5 & 0.215 & 0.481 & 0.304 & & 0.5 & 0.237 & 0.488 & 0.275 \\
\hline & & 2 & 0.172 & 0.411 & 0.209 & & & 0.191 & 0.427 & 0.191 & & & 0.213 & 0.438 & 0.175 \\
\hline & & 3 & 0.157 & 0.376 & 0.156 & & & 0.176 & 0.393 & 0.144 & & & 0.197 & 0.406 & 0.132 \\
\hline
\end{tabular}

the true values of unknowns. Another interesting result is that the value of $r_{j}^{*}$ decreases as $s_{\tau}$ increases. This implies that when the variation of unknowns gets large, the precision in estimation of systematic errors can become more desirable.

\section{Concluding remarks}

The results discussed in Sections 4 and 5 can be easily extended to the case that a cost constraint is considered in the measurement process. Suppose that each measurement of standards $S_{0}$ and $S_{1} \operatorname{cost} c_{0}$ and $c_{1}$ dollars, respectively, and each measurement of any unknowns costs $c$ dollars. Moreover, the total cost is assumed to be budgeted within $B$ dollars. The problem thus turns out to be in determination of $a_{0}, a_{1}$ and $n_{j}$, for $j=1,2, \ldots, m$, such that the objective function

$$
f\left(a_{0}, a_{1}, n_{1}, n_{2}, \ldots, n_{m}, \theta_{0}, \theta_{1}\right)=\frac{\theta_{1}}{a_{0}}+\frac{\theta_{0}}{a_{1}}+\sum_{j=1}^{m} \frac{1}{n_{j}},
$$

is minimized subject to the constraint 


$$
c_{0} a_{0}+c_{1} a_{1}+c \sum_{j=1}^{m} n_{j} \leq B .
$$

Similarly, we can obtain the following optimal allocation for the budget being exactly equal to $B$ dollars.

$$
\begin{aligned}
& a_{0}^{*}=\frac{B \sqrt{\theta_{1}}}{\sqrt{c_{0}}\left(\sqrt{c_{1} \theta_{0}}+\sqrt{c_{0} \theta_{1}}+m \sqrt{c}\right)}, \\
& a_{1}^{*}=\frac{B \sqrt{\theta_{0}}}{\sqrt{c_{1}}\left(\sqrt{c_{1} \theta_{0}}+\sqrt{c_{0} \theta_{1}}+m \sqrt{c}\right)}
\end{aligned}
$$

and

$$
n_{j}^{*}=\frac{B}{\sqrt{c}\left(\sqrt{c_{1} \theta_{0}}+\sqrt{c_{0} \theta_{1}}+m \sqrt{c}\right)}, \quad \text { for } j=1,2, \ldots, m .
$$

In practice, $a_{0}, a_{1}$ and $n_{j}$ can be taken to be the largest integers less than or equal to $a_{0}^{*}, a_{1}^{*}$ and $n_{j}^{*}$, respectively. Obviously, the optimal allocation still depends on $\tau_{1}, \tau_{2}, \ldots, \tau_{m}$. Therefore, the user still needs to use locally or Bayesian A-optimal measurements designs.

In this paper, we investigate construction of A-optimal measurement designs under the assumption that measurement errors are independent and have a homogenous variance in the linear calibration process. It is not uncommon to encounter situations where the measurements are serially correlated (Liao et al. 2000) or the variance of the measurement depends on its true value (Rocke and Lorenzato 1995). Certainly, the optimal allocation problem for the linear calibration process involving heterogeneous variances or correlated errors can become more complicated. We are currently investigating this interesting issue.

\section{Appendix}

The derivation of Equations (3.2), (3.3), (3.4) and (3.5)

After differentiating the log-likelihood of (3.1) with respect to $\alpha, \beta$ and $\tau_{j}$, for $j=1,2, \ldots, m$, we have the following.

$$
\begin{aligned}
& \sum_{i=1}^{N} y_{i}-N \hat{\alpha}-\left(a_{0} x_{0}+a_{1} x_{1}+\sum_{j=1}^{m} n_{j} \hat{\tau}_{j}\right) \hat{\beta}=0 \\
& \left(x_{0} \sum_{i=1}^{N} \delta_{i}^{S_{0}} y_{i}+x_{1} \sum_{i=1}^{N} \delta_{i}^{S_{1}} y_{i}+\sum_{j=1}^{m} n_{j} \bar{y}_{j} \hat{\tau}_{j}\right)-\left(a_{0} x_{0}+a_{1} x_{1}+\sum_{j=1}^{m} n_{j} \hat{\tau}_{j}\right) \hat{\alpha} \\
& \quad-\left(a_{0} x_{0}^{2}+a_{1} x_{1}^{2}+\sum_{j=1}^{m} n_{j} \hat{\tau}_{j}^{2}\right) \hat{\beta}=0
\end{aligned}
$$




$$
n_{j} \bar{y}_{j}-n_{j} \hat{\alpha}-n_{j} \hat{\tau}_{j} \hat{\beta}=0, \quad \text { for } j=1,2, \ldots, m .
$$

Clearly, Equation (3.4) results from (A.3). Then, we substitute (A.3) in (A.1) and (A.2) to yield

$$
\begin{aligned}
& \left(a_{0}+a_{1}\right) \hat{\alpha}+\left(a_{0} x_{0}+a_{1} x_{1}\right) \hat{\beta}=\sum_{i=1}^{N} \delta_{i}^{S_{0}} y_{i}+\sum_{i=1}^{N} \delta_{i}^{S_{1}} y_{i} \\
& \left(a_{0} x_{0}+a_{1} x_{1}\right) \hat{\alpha}+\left(a_{0} x_{0}^{2}+a_{1} x_{1}^{2}\right) \hat{\beta}=x_{0} \sum_{i=1}^{N} \delta_{i}^{S_{0}} y_{i}+x_{1} \sum_{i=1}^{N} \delta_{i}^{S_{1}} y_{i} .
\end{aligned}
$$

Finally, solving (A.4) and (A.5), we have Equations (3.2) and (3.3). Moreover, the expectation of the second derivatives of the log-likelihood are given by

$$
\begin{array}{ll}
-E\left[\frac{\partial^{2} l}{\partial \alpha^{2}}\right]=\frac{1}{\sigma^{2}} N, & -E\left[\frac{\partial^{2} l}{\partial \alpha \partial \beta}\right]=\frac{1}{\sigma^{2}}\left(a_{0} x_{0}+a_{1} x_{1}+\sum_{j=1}^{m} n_{j} \tau_{j}\right), \\
-E\left[\frac{\partial^{2} l}{\partial \alpha \partial \tau_{j}}\right]=\frac{1}{\sigma^{2}}\left(n_{j} \beta\right), & -E\left[\frac{\partial^{2} l}{\partial \alpha \partial \sigma^{2}}\right]=0, \\
-E\left[\frac{\partial^{2} l}{\partial \beta^{2}}\right]=\frac{1}{\sigma^{2}}\left(a_{0} x_{0}^{2}+a_{1} x_{1}^{2}+\sum_{j=1}^{m} n_{j} \tau_{j}^{2}\right), & -E\left[\frac{\partial^{2} l}{\partial \beta \partial \tau_{j}}\right]=\frac{1}{\sigma^{2}}\left(n_{j} \beta \tau_{j}\right), \\
-E\left[\frac{\partial^{2} l}{\partial \beta \partial \sigma^{2}}\right]=0, & -E\left[\frac{\partial^{2} l}{\partial \tau_{j}^{2}}\right]=\frac{1}{\sigma^{2}}\left(n_{j} \beta^{2}\right), \\
-E\left[\frac{\partial^{2} l}{\partial \tau_{j} \partial \sigma^{2}}\right]=0, & -E\left[\frac{\partial^{2} l}{\partial\left(\sigma^{2}\right)^{2}}\right]=\frac{N}{2 \sigma^{4}} .
\end{array}
$$

These elements constitute the Fisher information matrix. Then Equation (3.5) follows by some algebra calculations.

\section{References}

[1] Atkinson AC, Donev AN (1992) Optimum experimental designs. Oxford University Press, New York

[2] Brown PJ (1993) Measurement, regression and calibration. Oxford University Press, New York

[3] Fuller WA (1987) Measurement error models. John Wiley and Sons, New York

[4] Graybill FA (1983) Matrices with applications in statistics. Duxbury Press, Belmont, California

[5] Hunter WG, Lamboy WF (1981) A Bayesian analysis of the linear calibration problem. Technometrics 23:323-328

[6] Liao CT, Taylor CH, Iyer HK (2000) Optimal measurement designs when errors are correlated. J. Statist. Plann. Inference 84:295-321

[7] Pepper MPG (1973) A calibration of instruments with non-random errors. Technometrics 15:587-599

[8] Perng SK, Tong YL (1977) Optimal allocation of observations in inverse linear regression. Ann. Statist. 5:191-196

[9] Rocke DM, Lorenzato S (1995) A two-component model for measurement error in analytical chemistry. Technometrics 37:176-184 